\title{
Expression of bak in S. pombe results in a lethality mediated through interaction with the calnexin homologue Cnx1
}

\author{
Catherine N. Torgler ${ }^{1}$, Mariastella de Tiani ${ }^{1}$, Thomas Raven ${ }^{2}$, \\ Jean-Pierre Aubry ${ }^{1}$, Robin Brown ${ }^{2}$ and Eric Meldrum ${ }^{1,3}$ \\ 1 Geneva Biomedical Research Institute, chemin des Aulx 14, 1228 Plan-les- \\ Ouates Geneva, Switzerland. tel: 4122706 9666; fax: 4122794 6965; \\ e-mail: em13714@ggr.co.uk \\ 2 Cell Biology Unit, Glaxo Medicines Research Centre, Gunnels Wood Road, \\ Stevenage, Hertfordshire SG1 2NY, UK \\ 3 corresponding author: Geneva Biomedical Research Institute, chemin des Aulx \\ 14, 1228 Plan-les-Ouates Geneva, Switzerland. tel: 4122706 9666; fax: \\ 4122794 6965; e-mail: em13714@ggr.co.uk
}

Received 14.10.96; revised 12.12.96; accepted 16.12 .96

Edited by M. Piacentini

\begin{abstract}
Expression studies in the yeast S. pombe have been utilised to establish the basis for a genetic analysis designed to identify the lethal partners of the pro-apoptotic proteins bak and bax. Bak expression in $S$. pombe is lethal and this lethality is rescued by co-expression of bcl-2 or bcl- $\mathrm{x}_{\mathrm{L}}$. S. pombe cells expressing bak have a terminal phenotype in which the majority of cells are blocked in the G1 phase of the cell cycle while the remainder of cells, unable to complete M-phase, miscoordinate the timing of subsequent events in the cell cycle. Although bax expression in S. pombe gives rise to a slow growth phenotype, not a lethality, bax expressing cells display the same cell cycle phenotypes described for bak. Electron microscopy of cells expressing bak reveals a dramatic accumulation of large vesicular structures. A two-hybrid screen designed to identify $S$. pombe proteins which interact with bak, isolated the S. pombe calnexin homologue cnx1. Genetic analysis demonstrates that the $\mathrm{Cnx1}$ domain which binds to bak in two-hybrid experiments, is necessary for bak lethality in S. pombe. This report identifies a lethal interacting partner for bak and the observations suggest a model for bak mediated lethality which can be tested in higher cells.
\end{abstract}

Keywords: apoptosis, programmed cell death, Schizosaccharomyces pombe, bcl-2 family, bak, calnexin

Abbreviations: MM: minimal medium; NLM: nitrogen limiting medium; FACS: fluorescence activated cell sorting

\section{Introduction}

In higher eukaryotic cells an important control of the apoptotic programme is exerted by members of the bcl-2 family of proteins (Williams and Smith 1993; Reed 1994; Oltvai and Korsmeyer 1994). Different members of this family, on the basis of over-expression or gene knockout experiments, have the ability to commit the cell to, or protect the cell from, apoptosis (Garcia et al, 1992; Oltvai et al, 1993; Veis et al, 1993; Motoyama et al, 1995). Proteins of the bcl-2 family demonstrate a common ability to form homo and heterodimers, thus leading to a model where a cell's apoptotic fate is determined by competitive binding between lethal and protecting members of the family (Korsmeyer et al, 1993). However, the complexity of interactions amongst members of the bcl-2 family (Farrow and Brown 1996), coupled with the lack of any known biochemical activity for these proteins has hampered the identification of other components of the apoptotic pathway in higher cells. Consequently, fundamental questions remain concerning their mechanisms of action, in particular the identity of the cellular targets for the two known apoptotic inducers bak (Farrow et al, 1995; Chittenden et al, 1995; Kiefer et al, 1995) and bax (Oltvai et al, 1993).

$\mathrm{Bcl}-2$ family member homologues have been identified in evolutionarily distinct species suggesting that, at least at the level of these proteins, the apoptotic programme has been conserved throughout evolution. Indeed, only the genetic analysis of somatic cell death in the nematode $C$. elegans has established a linear apoptotic pathway involving a bcl-2 family member (Hengartner and Horvitz 1994; Ellis et al, 1991). Until recently, it was widely believed that the evolution of an 'altruistic' apoptotic pathway coincided with the emergence of multi-cellular organisms. This supposition however, failed to appreciate that many features regulated by apoptosis in multicellular organisms (i.e. cell number, differentiation state and growth arrest) are important features of the life cycle of many unicellular eukaryotes. Recently, several reports have indicated that an apoptosis-like process operates in several different unicellular eukaryotes, thus raising important new questions concerning the evolutionary origin of apoptosis (Cornillon et al, 1994; Ameisen et al, 1995; Wellburn et al, 1996, reviewed in Ameisen 1996).

These new insights have introduced the possibility that unicellular eukaryotes may be of utility as genetically tractable models for apoptotic death. Assessing the contribution that such a model could make however, may only be possible upon the exploitation of the genetic tractability of unicellular organisms to identify the endogenous molecular mediators of death. This, in turn, would allow the construction of testable models for apoptotic death in higher cells.

In light of the above, we have explored the possibility that the yeast $S$. pombe may be a model system for certain aspects of apoptotic death. In this report we have sought to determine whether the pro-apoptotic proteins bak or bax elicit a selectable phenotype upon expression in $S$. pombe. Such a phenotype need not, in our opinion, form the basis of a demonstration of classical apoptotic morphology but should establish the basis for a genetic analysis designed 
to identify the components of the bak/bax controlled pathway(s) in yeast. In this report we demonstrate that bak expression in $S$. pombe rapidly commits cells to a cell cycle specific lethality which is rescued by co-expression of bcl-2 and bcl- $x_{\mathrm{L}}$. In addition, we identify an $S$. pombe protein which interacts with bak upon two-hybrid analysis and demonstrate that this interaction is necessary for bak induced lethality in $S$. pombe.

\section{Results}

\section{Bak expression in S. pombe is lethal}

To evaluate the phenotype in $S$. pombe upon expression of the pro-apoptotic proteins bak and bax, cDNAs were subcloned into the $\mathrm{REP}_{5}$ multi-copy plasmid under the control of the thiamine repressible nmt 1 promoter (Maundrell 1990; Maundrell 1993) and transformed into a host S. pombe haploid strain (Materials and Methods). To asses cell division upon expression, growth was tested on solid MM lacking thiamine (Figure 1a). In the bax expressing strain growth was significantly slower than the control, while upon bak expression no growth was observed.

A time course of cell density was performed in order to gain a more detailed understanding of cell behaviour upon bak or bax expression (Figure 1b). After $20 \mathrm{~h}$ of logarithmic growth in MM lacking thiamine $(2 \mathrm{~h}$ after maximal mRNA expression was achieved see Figure 1c), the growth rate of cultures expressing bak or bax decreased. This difference corresponds to a rate of growth of one division every $9 \mathrm{~h}$ compared to the wild-type rate of one division every three and a half hours. After $60 \mathrm{~h}$, growth of the bak expressing culture had essentially ceased while the bax expressing culture, which exhibits the same time-course of mRNA induction as the bak culture (data not shown), continued to grow at the compromised rate.

To determine whether bak expression results in reversible cell cycle arrest or lethality, the extent to which the phenotype upon bak expression could be reversed by inhibiting the $n m t 1$ promoter with thiamine, was assessed (Figure 2). After $14 \mathrm{~h}$ of growth in the absence of thiamine, at which time bak mRNA is first detected (Figure 1c), cells first began to commit to death and after $18 \mathrm{~h} 100 \%$ of the cells became committed to die. Since the $n m t 1$ promoter is fully repressed by thiamine within $3 \mathrm{~h}$ (Maundrell 1990) and since the assessment of death is made 14 days after the addition of thiamine to the cells, we conclude that bak is lethal in S. pombe and that once bak expression is initiated, cells rapidly pass a critical point beyond which commitment to death is irreversible.

\section{Bak lethality is rescued by co-expression of bcl-2, bcl- $\mathrm{x}_{\mathrm{L}}$ and $\mathrm{E} 1 \mathrm{~B}$ 19K}

Protection from the bax induced slow growth and bak lethality described above is provided by co-expression of bcl-2 or bcl$\mathrm{x}_{\mathrm{L}}$, while only bak induced lethality is rescued by coexpression of the adenovirus E1B 19K protein (data not shown). The most simple explanation for these results is that protection from lethality is a consequence of heterodimer formation between bcl-2 or bcl- $x_{L}$ and bak or bax leading to inactivation. The apparent specificity of E1B $19 \mathrm{~K}$ rescue most likely reflects a difference in affinity for bak and bax.
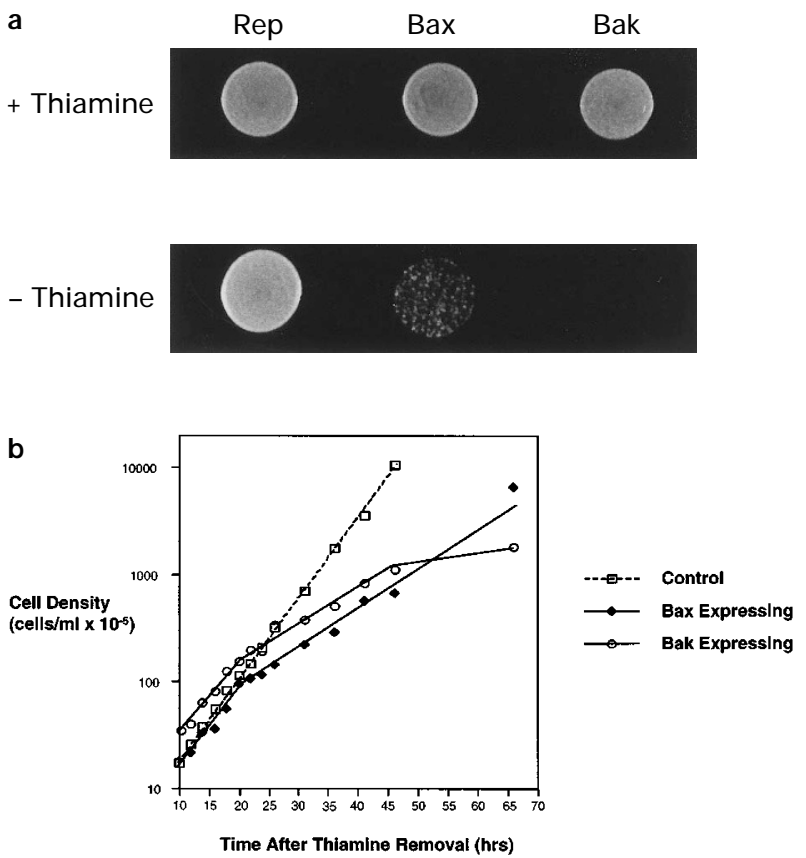

c Time After Thiamine

Removal (hours)

$\begin{array}{llllllllll}10 & 12 & 14 & 16 & 18 & 20 & 22 & 26 & 28 & 30\end{array}$

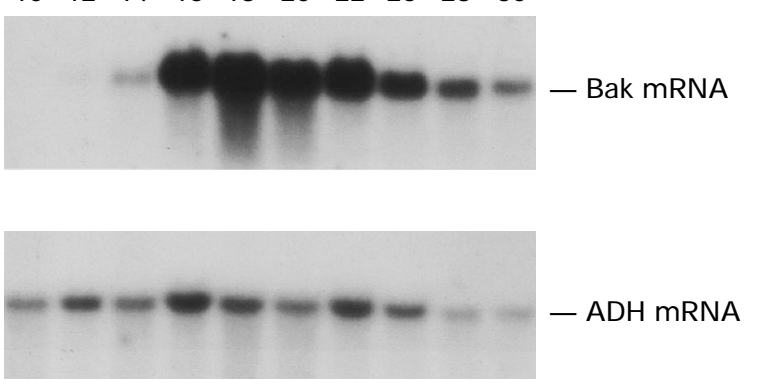

Figure 1 Effect of bak or bax expression upon growth rate in $S$. pombe. (a) $S$. pombe transformed with $R E P_{5}, R P_{5}$ : bax or REP : bak was grown for $20 \mathrm{~h}$ in liquid MM either lacking thiamine to induce expression or containing $4 \mu \mathrm{M}$ thiamine for controls. Cell concentrations were estimated with a haemocytometer and a volume of $20 \mu \mathrm{l}$, containing 100000 cells, was deposited onto MM plates either with $4 \mu \mathrm{M}$ thiamine (in the case of controls) or without thiamine. Photographs were taken after $48 \mathrm{~h}$ of growth at $30^{\circ} \mathrm{C}$. (b) S. pombe transformed with $\operatorname{REP}_{5}(-\cdots-\cdots)$, REP R $_{5}$ bax $(-\downarrow)$ or REP R $_{5}$ bak $(-\bigcirc-)$ was grown to logarithmic phase in MM containing $4 \mu \mathrm{M}$ thiamine. At the zero time point cells were washed three times with MM lacking thiamine and grown in the same media at $30^{\circ} \mathrm{C}$. To maintain the cells in logarithmic growth phase, cultures were regularly diluted and each dilution was taken into account for graphic representation of cell density on the $y$ axis so that the slope of the line is representative of rate of growth. (c) Time points for Northern analysis were taken from an experiment performed as described for Figure 1b. At the time points indicated, total RNA was isolated from $10^{8}$ cells and Northern blot analysis was performed as described in Materials and Methods. The same nylon filter was probed with bak cDNA and, as a control for sample loading, alcohol dehydrogenase (ADH) CDNA. 


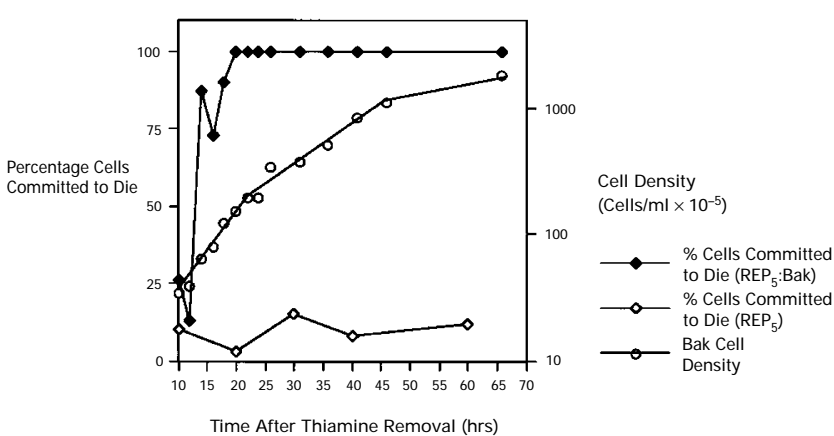

Figure 2 Bak expression rapidly commits $S$. pombe cells to death. S. pombe cells transformed with $\mathrm{REP}_{5}$ : bak or $\mathrm{REP}_{5}$ (as control) were grown in liquid MM lacking thiamine. At different time points after thiamine removal from the culture media, cells were removed from the culture and spread onto solid MM containing $4 \mu \mathrm{M}$ thiamine. After 14 days at $30^{\circ} \mathrm{C}$ the percentage cells committed to die was defined as the number of colonies which did not grow relative to the total number of cells spread. The growth curve of bak expressing cells from Figure $1 b$ is shown to ease comparison.

Heterodimer formation has been demonstrated for all these interactions by in vivo and in vitro experiments (Farrow and Brown 1996), although it is possible that rescue is due to competing pathways in yeast influenced by $b c l-2$, bcl- $x_{L}$ or E1B 19K expression. It should be noted that while the expression of bak or bax elicits observable phenotypes in $S$. pombe the expression of the bcl-2 family members which have apoptotic protective functions in higher cells, such as bcl-2 or bcl- $x_{\mathrm{L}}$, elicit no detectable growth effect when expressed alone.

\section{Bak expression in S. pombe provokes a cell cycle specific lethality}

To gain an understanding of the nature of the lethality caused by bak expression, an analysis of the cellular DNA content and morphology upon expression was performed (Materials and Methods). DNA content was measured by flow cytometry analysis on $\mathrm{REP}_{5}$ : bak transformed cells taken at different time points after thiamine removal from the culture media (Figure 3). When maximal bak mRNA expression was achieved (18 h, see Figure 1c) all the cells in the population had the $2 n$ DNA content characteristic of logarithmically growing $S$. pombe. By $26 \mathrm{~h}$, cells with a 1n DNA content began to appear and the proportion of $1 \mathrm{n}$ cells increased with time until the culture had ceased dividing. By $60 \mathrm{~h}, 83 \%$ of cells in the culture had a 1n DNA content. It should be noted that the DNA content of control cells was not affected by long term growth in the absence of thiamine. These results suggest that bak expression in exponentially growing $S$. pombe hinders progression into S-phase of the cell cycle so that the terminal phenotype of the majority of cells is in G1.

Microscopic analysis of cells stained with Hoechst (nuclear stain) and Calcofluor (septal stain) (Materials and Methods) demonstrated that, although all of the bak expressing cells are irreversibly committed to die (see Figure 2), approximately $90 \%$ of expressing cells had a
A

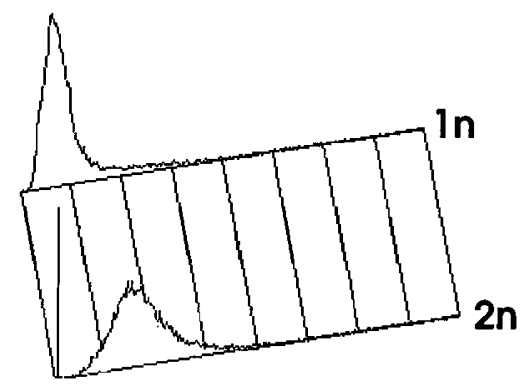

B

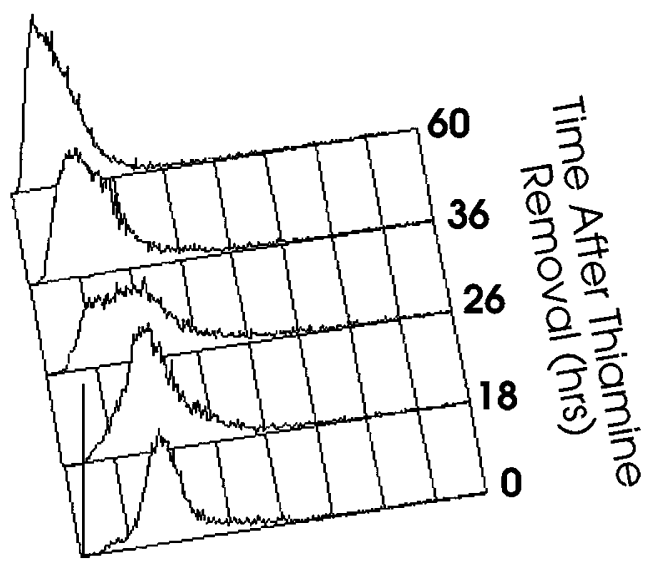

Figure 3 Bak expression leads to an accumulation of cells with a 1n DNA content. (a) As a 1n DNA content control, S. pombe was grown to stationary phase in nitrogen-limited medium (NLM) and flow cytometry analysis performed. The $2 n$ DNA content control was a wild-type logarithmically growing $S$. pombe culture. (b) $S$. pombe transformed with $R_{E} P_{5}$ : bak was grown in MM lacking thiamine to induce bak expression and the DNA content of the cells at the times shown after thiamine removal measured by flow cytometry analysis. In both graphs shown, the x-axis represents fluorescence as a measure of DNA content and the third dimension axis is representative of cell number.

morphology similar to that which is found in wild-type cultures except for a slightly larger length and diameter. Cells with a distinctive morphology not found in wild-type cultures however, began to appear $20 \mathrm{~h}$ after thiamine removal from the culture (Figure 4). Cells with an asymmetric nuclear distribution across the axis of the septum are observed upon bak expression (Figure 4b, c, d and $h$ ) and these cells are capable of cytokinesis (Figure $4 d)$ giving rise to anucleate cells (Figure 4e). Cells with brightly staining septa (Figure $4 b, c, f, g$ and h), multiple septa (Figure $4 f$ and $g$ ), septa which deviate from the perpendicular axis of the cell (Figure $4 \mathrm{~b}$ and $\mathrm{h}$ ) and abnormally elongated cells (Figure $4 \mathrm{i}$ ) are also observed upon bak expression. The proportion of these phenotypes, found in a bak expressing culture is shown in Table 1.

In order to determine DNA content of the aberrant cells shown in Figure 4, cells expressing bak after $36 \mathrm{~h}$ growth in the absence of thiamine (1:1 ratio of $1 n: 2 n$ see Figure 3 ) were separated by FACS into a $1 n$ and $2 n$ population. Microscopic analysis of these two populations stained with Hoechst and Calcofluor revealed that the phenotypes shown in Figure 4 were found only in the $2 n$ population. 
These cells appear therefore to have overcome the G1 cell cycle block imposed by bak expression, completed Sphase and failed to complete M-phase. They remain either in the G2 growth phase of the cell cycle giving rise to abnormally long cells, or begin to mis-coordinate the timing of cell-cycle events with septation and cytokinesis a
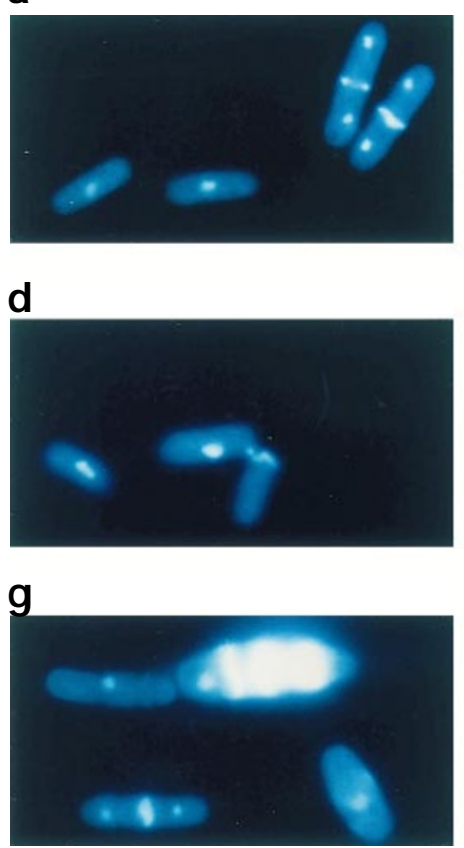

b

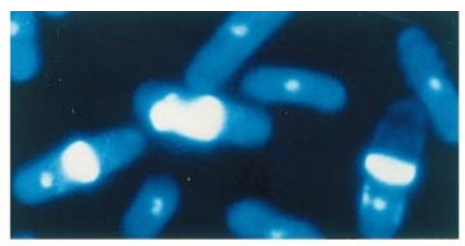

e

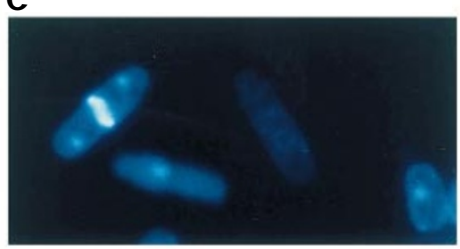

h

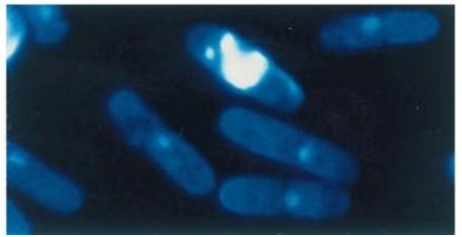

C
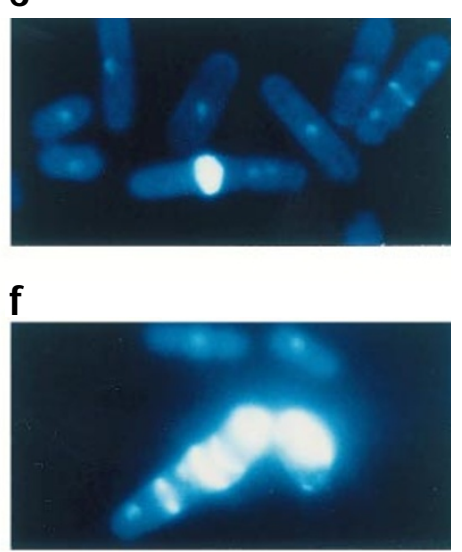

i

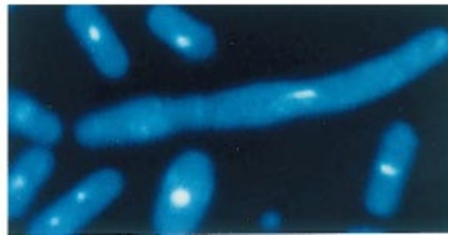

Figure 4 Expression of bak in $S$. pombe provokes a characteristic phenotype. S. pombe transformed with REP : bak was grown in MM lacking thiamine for $42 \mathrm{~h}$ and stained with Hoechst (nuclear stain) and Calcofluor (septal stain). Wild-type cells (a) are shown for comparison with bak expressing cells (b to i). Cells with an assymetric nuclear distribution across the septum are shown (b, c, $\mathbf{d}$ and $\mathbf{h})$. Cells with an intense septal staining (b, c, $\mathbf{f}, \mathbf{g}, \mathbf{h})$, septum deviated from the perpendicular axis of the cell (b and $\mathbf{h}$ ) and multiple septated cells ( $\mathbf{f}$ and $\mathbf{g})$ are shown. Also shown is an abnormally elongated cell (i) a cytokinesing cell which has an assymetric nuclear distribution across the septum (d) and an anucleate cell (e). All photographs were taken with a magnification of $1000 \times$.

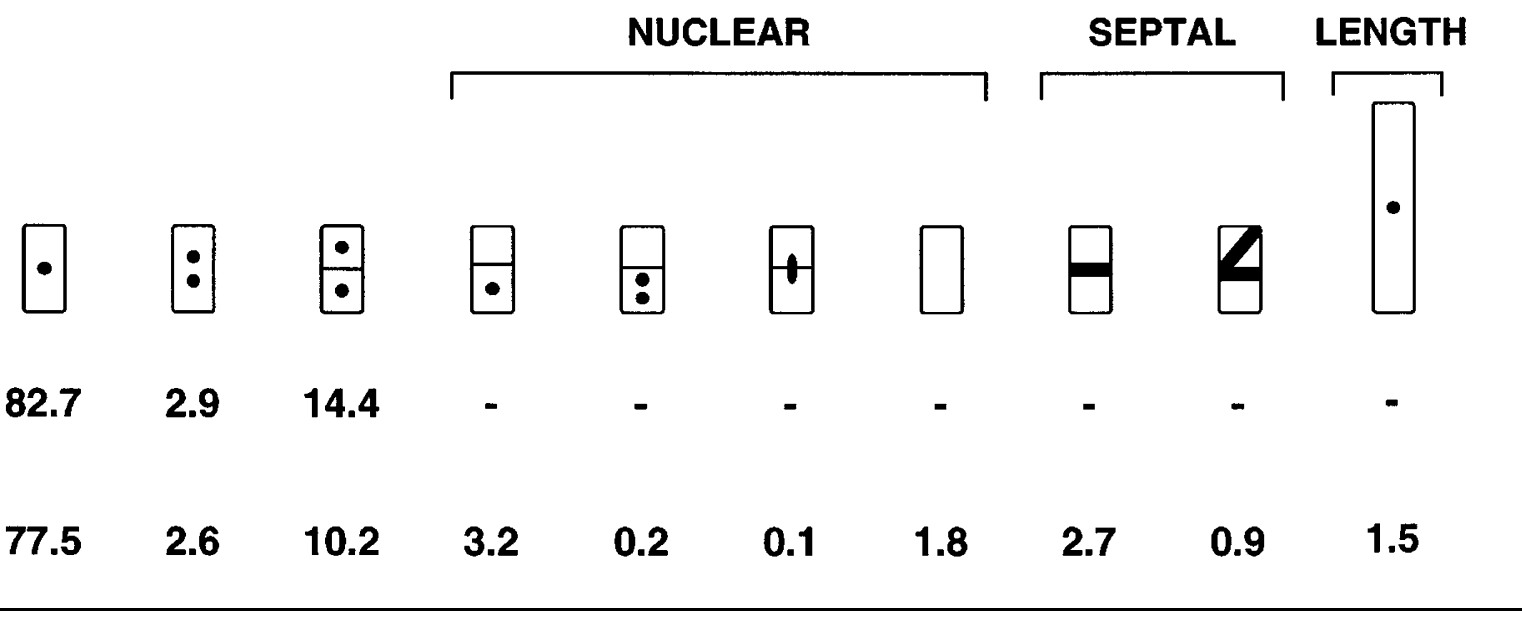

Wild-type S. pombe and S. pombe transformed with $\mathrm{REP}_{5}$ : bak were maintained at logarithmic growth phase in MM lacking thiamine for $60 \mathrm{~h}$ and stained with Hoechst and Calcofluor. For both experimental subjects a total of 3000 cells were counted and orgainsed into the phenotypes diagrammatically shown. It should be noted that for simplicity, in each abnormal phenotypic category (i.e. abnormal nucleus, septum or length) only one single characteristic is expressed as a percentage and represented in each cellular diagram. As a consequence there is overlap between the phenotypes and for the cells transformed with REP ${ }_{5}$ : bak the total percentage is greater than $100 \%$ 
occurring before M-phase completion and repeated septum deposition occurring, sometimes in an incorrect orientation.

A

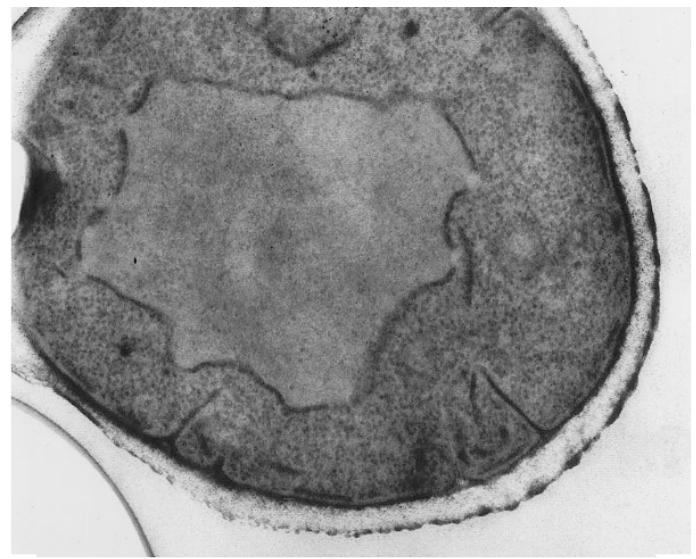

B

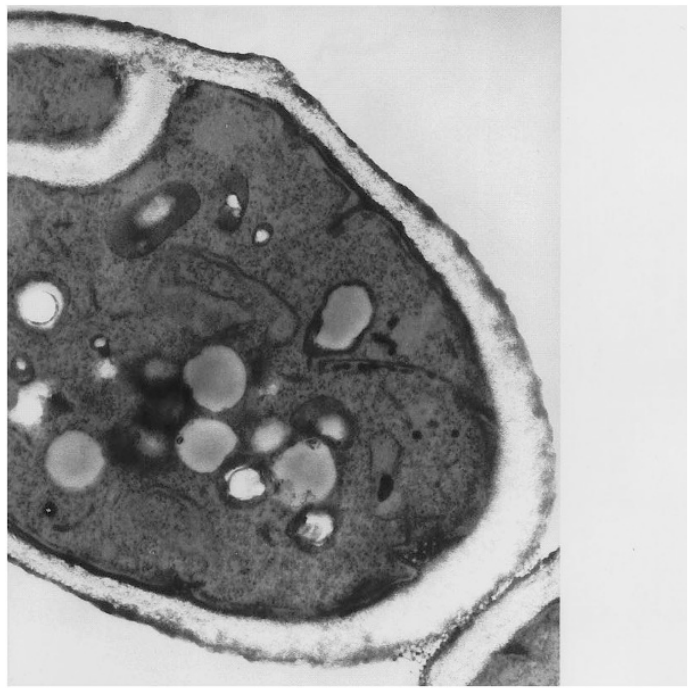

C

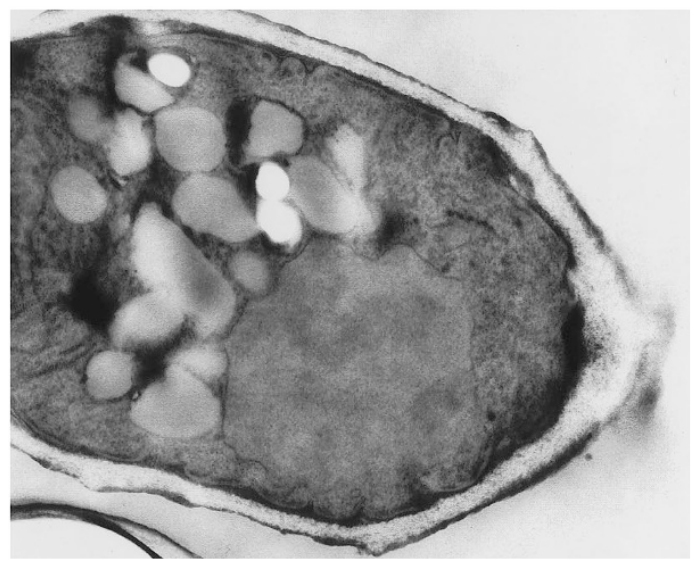

Figure 5 Bak expression in $S$. pombe results in the accumulation of large vesicular structures. S. pombe cells transformed with $\mathrm{REP}_{5}$ as control (a) or $\mathrm{REP}_{5}$ : bak (b and $\mathbf{c}$ ) were grown for $42 \mathrm{~h}$ in liquid MM lacking thiamine. Cells were then fixed and treated as described in Materials and Methods.
Similar analysis of bax expressing cells demonstrated the presence, in comparable proportions, of all the aberrant phenotypes described for bak. In addition, upon bax expression the percentage of cells with a 1n DNA content was elevated relative to the control (approximately $20 \%$ compared to $8 \%$ for control - results not shown) but $1 \mathrm{n}$ cells did not accumulate with prolonged expression.

In order to examine more closely the morphology of bak expressing cells in comparison with wild type $S$. pombe both cultures were analyzed by electron microscopy. The most striking change upon bak expression was the appearance, in all the expressing cells analyzed, of large vesicular structures (Figure $5 b$ and $c$ ). In the majority of bak expressing cells the nuclei appeared to have normal morphology. After a long period of bak expression in $S$. pombe $(72 \mathrm{~h})$ genomic DNA degradation can be observed on agarose gels but this is unlikely to be a primary determining factor in the bak-induced cell death, since commitment to death occurs very rapidly upon induction of bak expression (See Figure 2).

\section{Bak lethality is mediated in part through interaction with $\mathrm{Cnx1}$}

In order to determine whether the bak induced lethality was dependant on the physical interaction of bak with an S. pombe protein and to isolate this protein, an $S$. pombe two-hybrid cDNA library (Clontech) was screened with human bak protein fused to GAL-4 as 'bait'. Ten million cDNA clones were screened and 31 positives were isolated that interacted with the bak protein and not with a range of non-specific baits. These were extracted and sequenced. All 31 positives were GAL4 fusions with the $S$. pombe cDNA encoding the calnexin homologue Cnx1 (Figure 6a) which is an ER integral membrane protein with a cytosolically directed C-terminus (Parlati et al, 1995). Calnexin is an ER protein expressed in all eukaryotic cells which functions as a molecular chaperone and as a component of the ER quality control apparatus (Bergeron et al, 1994). This positive was of particular interest as bak and other bcl-2 family members are known to colocalise (in higher cells) with calnexin in the ER (Chen-Levy et al, 1989; Jacobson et al, 1993 and R. Brown unpublished). The smallest clone isolated contained only the C-terminal 99 amino acids of $\mathrm{Cnx} 1$, sufficient to code for the transmembrane and C-terminal tail of the protein. No other members of the bcl2 family, with the exception of bax, demonstrated a positive interaction with $\mathrm{Cnx} 1$ when tested by two-hybrid analysis. In the case of bax the interaction was significantly weaker than that seen with bak, being only just above the background for detection. This difference in affinity for $\mathrm{Cnx} 1$ may underlie why bak and bax appear to elicit the same phenotype with different severities.

In order to test whether interaction between the cytosolic domain of $\mathrm{Cnx} 1$ and bak is responsible for lethality in $S$. pombe a strain deleted for wild type $c n x 1$ was utilised. The normally lethal effect of $c n x 1$ deletion was averted by expression of either the wild-type $c n x 1$ gene or a C-terminal truncated version which terminates at amino acid 524. This truncated Cnx1 still inserts into the ER membrane and complements deletion of the wild-type gene fully (Parlati et 
al, 1995) but does not contain the cytosolic domain which binds bak in two-hybrid experiments. While both the strains utilised were equally viable, the presence or absence of the cytosolic domain of $\mathrm{Cnx} 1$ altered the phenotype observed upon bak expression. Bak was lethal in the strain expressing wild-type $\mathrm{Cnx} 1$ but not in the strain expressing $\mathrm{C}$-terminal truncated $\mathrm{Cnx} 1$ (Figure $6 \mathrm{~b}$ ). Although deletion of the cytosolic domain of Cnx1 was sufficient to allow cells to overcome bak induced lethality it can be seen from Figure $6 \mathrm{~b}$ that growth is still slower than controls. It is not clear whether this compromised growth rate is representative of the doubling time of the cells or whether it is representative

\section{A}

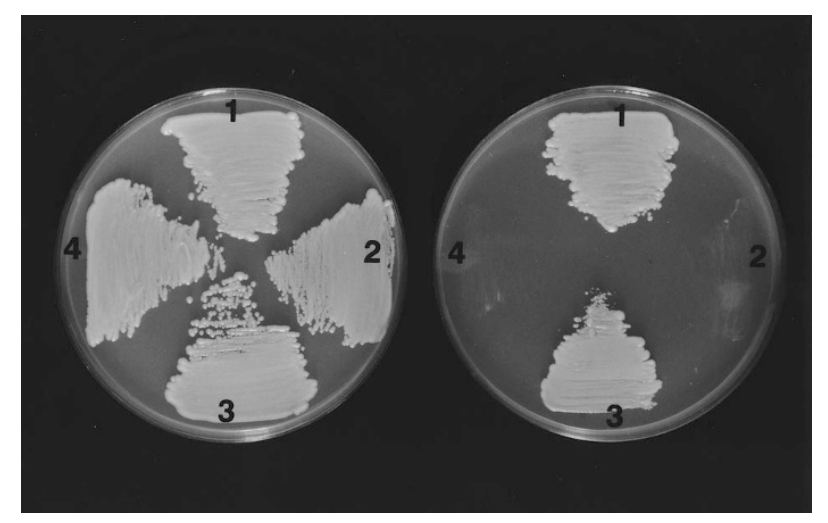

B

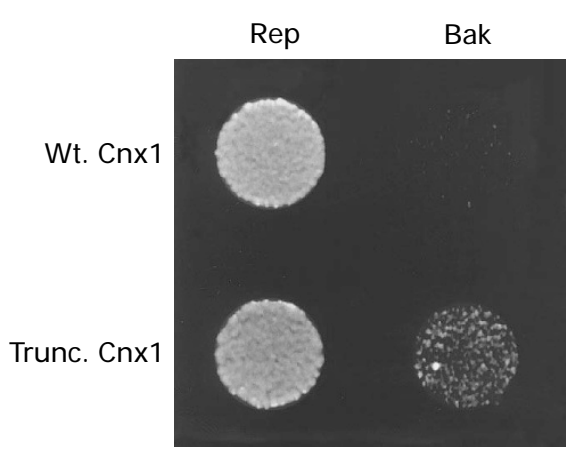

Figure 6 Bak interaction with $\mathrm{Cnx} 1$ is necessary for lethality in $S$. pombe. (a) In order to demonstrate the two hybrid interaction between bak and $\mathrm{Cnx} 1$ the S. cerevisiae strain Y190 was transformed with a plasmid containing the full length bak cDNA fused to the binding domain of the yeast GAL4 protein. This strain was then transformed with a vector containing the yeast GAL4 DNAbinding domain fused to either the $c n \times 1, b c l-x$ or bax cDNAs. These strains were then streaked onto plates containing either selective medium alone (left plate) or selective medium with the addition of $25 \mathrm{mM} 3$-aminotriazole (3-AT), right plate. A GAL4-dependant trans-activation of the HIS gene promoter in these strains permits growth in the presence of 3-AT and demonstrates a positive interaction. Both plates are divided into 4 sectors. Sector 1=bak vs. cnx1, Sector 2=bak vs. GAL4 activation domain (negative control), Sector $3=$ bak vs. bcl-x (positive control), Sector 4=bak vs. bax (negative control). Interaction of bak with $\mathrm{Cnx} 1$ and bcl-x in this two hybrid experiment also resulted in strong lac-z staining confirming the interaction with the second assayable marker (data not shown). (b) The cells shown are deleted for the $S$. pombe $c n x 1$ gene function and viability is maintained by expression of either wild type $\mathrm{Cnx}_{1}$ or a truncated $\mathrm{Cnx} 1$ which terminates at amino acid 524. These two strains were transformed with $\mathrm{REP}_{5}$ or $\mathrm{REP}_{5}$ : bak and grown for $20 \mathrm{~h}$ in liquid MM lacking thiamine to induce expression. Cell concentrations were estimated with a haemocytometer and a volume of $20 \mu$ l, containing 50000 cells was deposited onto an MM plate lacking thiamine. The photograph was taken after $48 \mathrm{~h}$ of growth at $30^{\circ} \mathrm{C}$ of a Gaussian distribution of severity of phenotype in the population.

\section{Interaction between bak and $\mathrm{Cnx} 1$ results in a dominant lethal effect}

A simple model to explain why the association of bak and Cnx1 contributes to lethality in S. pombe is that the interaction blocks normal Cnx1 function. If this were the case, bak induced lethality in a wild-type $S$. pombe genetic background should be rescued by the overexpression of either wild-type or $\mathrm{C}$-terminally truncated forms of the $\mathrm{Cnx}-1$ protein. No rescue from bak lethality in $S$. pombe was observed upon overexpression of either of these two forms of calnexin (data not shown). While the failure of full length Cnx1 to rescue bak lethality might be explained by insufficient levels of $\mathrm{Cnx1}$ protein, this cannot be the case for the C-terminally truncated Cnx1 since it does not contain the bak binding domain but does fully complement the lethal $c n x 1$ gene deletion (Parlati et al, 1995). The failure to rescue bak lethality, in a wild-type cnx 1 background, by overexpression of two different forms of Cnx1 suggests that the interaction between bak and $\mathrm{Cnx1}$ results in a dominant lethal effect perhaps due to the propagation of a lethal signal or the recruitment of additional interactors to the bak Cnx1 complex.

\section{Discussion}

Overexpression of the killing Bcl-2 family members bak and bax has been shown to be lethal across a diverse selection of evolutionarily distinct cell types ranging from humans, $C$. elegans and now the yeast $S$. pombe. In addition, across this same evolutionary distance the protecting family members $\mathrm{bcl}-2$ and bcl- $\mathrm{x}_{\mathrm{L}}$ have been shown to protect from that lethality. Bak expressing $S$. pombe cells have a terminal phenotype in which the majority of cells are arrested in the G1 phase of the cell cycle while the remainder of cells, unable to complete $M$ phase, mis-coordinate the timing of subsequent events in the cell cycle such as septation and cytokinesis. In bax expressing $S$. pombe, cells enter S-phase less efficiently than wild type and also mis-coordinate post M-phase events. Bak and bax appear to differ therefore only in the severity of phenotype upon expression, thus suggesting that bak and bax have at least one common mode of action in S. pombe.

Electron microscopy analysis of cells expressing bak reveals a dramatic accumulation of large vesicular structures. Intriguingly, similar morphological changes have been observed in Dictyostelium cells stimulated, with physiological inducers, to undergo a programmed cell death (Cornillon et al, 1994), in C. elegans cell death (Robertson and Thomson 1982) and in some instances of higher eukaryote cell death (Clarke 1990). It remains to be seen whether this cellular feature is a consequence of a common molecular mechanism of death.

A two-hybrid screen designed to identify $S$. pombe proteins which interact with bak, isolated the $S$. pombe calnexin homologue $c n x 1$. Genetic analysis demonstrates that the Cnx1 domain which binds to bak in two-hybrid experiments, is necessary for bak lethality in $S$. pombe. These results strongly suggest that bak lethality in $S$. 
pombe is, at least in part, caused by the interaction between bak and the cytosolic domain of $\mathrm{Cnx} 1$. Since bak expression in a genetic background deleted for the $\mathrm{Cnx} 1$ cytosolic domain still results in a compromised growth rate, it is likely that the lethal phenotype in $S$. pombe is driven by the interaction of bak with multiple proteins and that only one of those lethal interactions is with Cnx1. Interestingly, bax also interacts with $\mathrm{Cnx} 1$ in two-hybrid experiments but with significantly less affinity than bak. This difference in affinity for $\mathrm{Cnx} 1$ perhaps underlies why bak and bax appear to elicit the same phenotype with different severities.

Attempts to protect from bak lethality in $S$. pombe by coexpression of full length and C-terminally truncated $\mathrm{Cnx1}$ were unsuccessfull thus suggesting that bak lethality is not due to a recessive loss of Cnx1 function upon bak binding. Support for this is further provided by the observation that the phenotype associated with $c n x 1$ gene disruption in $S$. pombe is not similar to the bak expression phenotype described in this report (E. Meldrum unpublished). It therefore appears that the bak $\mathrm{Cnx} 1$ interaction provokes a dominant lethal effect perhaps due to the propogation of a lethal signal or the recruitment of additional interactors to the bak Cnx1 complex. The nature of this dominant lethality is currently under investigation by classical genetic approaches to generate bak resistant strains, by $c n x 1$ mutagenesis and by biochemical analysis of $\mathrm{Cnx} 1$ function in bak expressing cells.

In our opinion, the significance of bak lethality in $S$. pombe would be emphasised if it was possible to abrogate the same cellular functions by expressing another proapoptotic protein, not in the bcl-2 family but on the same programmed death pathway. This has been tested with the $C$. elegans pro-apoptotic protein Ced-4. Expression of Ced4 in $S$. pombe induces a lethality qualitatively similar to that described in this report i.e. accumulation of $1 \mathrm{n}$ cells $(50 \%$ after $50 \mathrm{~h}$ growth in the absence of thiamine) and mistiming of post M-phase events in the cell cycle to give rise to phenotypes shown in Figure 4. The nature of this Ced-4 lethality suggests that, even though they possess no sequence similarity, bak and Ced-4 interact with the same cellular function in $S$. pombe. The mechanisms of Ced-4 lethality in $S$. pombe are currently under investigation.

When one considers the evolutionary distance across which killing members of the bcl-2 family have now been shown to be lethal one has to propose that, unless bak is killing in these diverse cell types by chance alone, bak is interacting with a highly conserved cellular target or function. While yeast does not appear to possess an apoptotic programme, it cannot be discounted that one or more pathways in yeast may have been adapted by higher eukaryotes to provide an apoptotic pathway or alternatively, that bak may abrogate the same highly conserved cellular function in both yeast and higher eukaryotes. It is consistent with this reasoning to report that bak expression in $S$. pombe interferes with normal cell cycle progression, as components which regulate cell cycle progression are highly conserved across evolution (Morgan 1995). In addition, it is demonstrated that bak induced lethality in $S$. pombe is achieved in part through the interaction between bak and the ER localised calnexin homologue $\mathrm{Cnx} 1$. There is $50 \%$ sequence homology and a large degree of functional conservation between the human and $S$. pombe calnexin proteins. Bak in $S$. pombe appears therefore to be interacting with highly conserved cellular functions thus suggesting that the same bak lethal targets may be conserved between $S$. pombe and higher cells.

Bcl-2 family members in higher cells are known to be localised in the mitochondria, nuclear envelope and colocalised with calnexin at the ER (Gonzalez-Garcia et al, 1994; Krajewski et al, 1993; Chen-Levy et al, 1989; Jacobson et al, 1993), and it appears that the precise subcellular localisation of their function is cell type dependant (Zhu et al, 1996). It remains to be seen whether the highly conserved calnexin function also interacts with bak protein in higher cells. If so then this interaction may uncover part of the mechanism by which bak, resident in the ER (R. Brown unpublished), elicits its lethality. This analysis in higher cells, and the identification of the additional mechanisms by which bak provokes lethality in $S$. pombe, will be of central importance in the final assessment of bak mediated lethality in $S$. pombe as a model system for studying bcl-2 family member function.

The data described in this report establish the basis for genetic screening for the targets of bak/bax lethality in $S$. pombe. The observation that the $S$. pombe calnexin homologue $\mathrm{Cnx} 1$ is one of the lethal partners of bak, represents the first identification of a lethal partner of bak in any system. Further genetic analysis of the additional mechanisms of bak lethality in $S$. pombe will permit the identification of all the targets of bak action in $S$. pombe, and thus uncover additional functional principles for bcl-2 family members which can be tested in higher eukaryote cells.

\section{Materials and Methods}

\section{Strains and culture media}

All experiments described utilised the haploid $S$. pombe strain Sp30 $\left(h^{-}\right.$-leu1-32 ura4-D18 ade6-704 his5-303) based on the original wild-type $975 \mathrm{~h}^{+}$strain from the Berne collection. All strain constructions were performed by standard genetic procedures. All experiments were carried out with cells in logarithmic growth phase $\left(<1 \times 10^{7}\right.$ cells $\left./ \mathrm{ml}\right)$. Minimal medium (MM) used for liquid cultures or for plates had the same composition as described (Moreno et al, 1991), except that the $\mathrm{NH}_{4} \mathrm{Cl}$ concentration was at $2.5 \mathrm{~g} /$ /itre instead of $5 \mathrm{~g} /$ litre and the appropriate amino-acids were omitted in order to ensure plasmid maintenance. Nitrogen limiting media (NLM) had the same composition as $\mathrm{MM}$, except that the $\mathrm{NH}_{4} \mathrm{Cl}$ concentration was at $267 \mathrm{mg} /$ /itre. To induce the $\mathrm{nmt1}$ promoter, cells grown in liquid MM containing $4 \mu \mathrm{M}$ thiamine were washed three times with $10 \mathrm{ml}$ of MM lacking thiamine and cultured in this medium. In all experiments, cell densities were determined with a haemocytometer.

\section{Northern analysis}

Total mRNA was isolated as previously described (Elder et al, 1983) and separated electrophoretically on a $1 \%$ agarose gel containing 2.2 M formaldehyde (Sambrook et al, 1989). RNA was transferred to $\mathrm{N}$-Hybond nylon filters, and hybridised for $12 \mathrm{~h}$ with a ${ }^{32} \mathrm{P}-\alpha-\mathrm{dCTP}$ random labelled probe in $50 \%$ formamide buffer at $42^{\circ} \mathrm{C}$. Three 
washes in $1 \times \mathrm{SSC}, 0.1 \% \mathrm{SDS}(\mathrm{w} / \mathrm{v})$ at room temperature and a single wash with $0.1 \times \mathrm{SSC}, 0.1 \% \mathrm{SDS}(\mathrm{w} / \mathrm{v})$ for $90 \mathrm{~min}$ at $60^{\circ} \mathrm{C}$ were performed and blots were exposed for $44 \mathrm{~h}$ at $-70^{\circ} \mathrm{C}$ with an intensifying screen.

\section{FACS analysis}

For each time point, $1 \times 10^{7}$ cells were collected by centrifugation at $1000 \times g$ for $5 \mathrm{~min}$, washed three times in PEM (100 mM PIPES (sodium salt), $1 \mathrm{mM} \mathrm{EGTA,} 1 \mathrm{mM} \mathrm{MgSO}_{4}$ ) and resuspended in $200 \mu \mathrm{l}$ of PEM. Half of this cell suspension was Hoechst stained by the addition of $2 \mu \mathrm{l}$ of a $6 \mathrm{mg} / \mathrm{ml}$ frozen stock solution followed by a $30 \mathrm{~min}$ incubation at $37^{\circ} \mathrm{C}$. Cells were then fixed in $4 \mathrm{ml}$ of $2 \%(\mathrm{v} / \mathrm{v})$ Paraformaldehyde and stored in the dark at $4^{\circ} \mathrm{C}$. Flow cytometry was performed with a FACStar plus (Becton Dickinson, Erembodegem, Belgium) equipped with a UV argon ion laser (Spectra Physics, Les Ullis, France) emitting $120 \mathrm{~mW}$ at $351-364 \mathrm{~nm}$. The blue fluorescence of Hoechst 33342 was measured through a $450 / 10$ band pass filter. 10000 cells were recorded for each time point and Hoechst fluorescence was displayed on a linear scale with the pulse area measured using the pulse processing module.

\section{UV microscopy}

$5 \times 10^{7}$ cells were centrifuged at $1000 \times \mathrm{g}$ for $5 \mathrm{~min}$. Cell pellets were washed twice with PEM (see above) and resuspended in $200 \mu \mathrm{l}$ of PEM containing Calcofluor. The Calcofluor solution was prepared immediately before use by dissolving $5 \mathrm{mg}$ of Calcofluor in $1 \mathrm{ml}$ of PEM. Undissolved material was removed by centrifugation at $10000 \times \mathrm{g}$ for $5 \mathrm{~min}$. The cells were resuspended in $200 \mu \mathrm{l}$ of a fivefold dilution of the Calcofluor supernatant prepared above, then incubated on a rotary inverter in the dark at room temperature for $15 \mathrm{~min}$. Cells were washed three times with, and finally resuspended in, $1 \mathrm{ml}$ of PEM. For nuclear staining, $2 \mu \mathrm{l}$ of a $6 \mathrm{mg} / \mathrm{ml}$ frozen stock solution of Hoechst was added to $100 \mu \mathrm{l}$ of cells stained with Calcofluor as described above. Cells were incubated for $30 \mathrm{~min}$ at $37^{\circ} \mathrm{C}$. Cells were mixed with an equal volume of antifade (Molecular probes) and after vortexing, $3 \mu \mathrm{l}$ was applied to a microscope slide, a coverslip applied and sealed with nail polish. Cells were viewed with a fluorescence microscope (Zeiss) using a UV filter (487702 A).

\section{Electron microscopy}

S. pombe cells transformed with $\mathrm{REP}_{5}$ or $\mathrm{REP}_{5}$ : bak were grown for $42 \mathrm{~h}$ in liquid $\mathrm{MM}$ lacking thiamine. Cells were then fixed with Karnovsky fixative (2\% paraformaldehyde and $2.5 \%$ glutaraldehyde in $0.1 \mathrm{M}$ sodium cacodylate) for $15 \mathrm{~min}$ at $20^{\circ} \mathrm{C}$ followed by post-fixation in $1 \%$ osmium tetroxide in $0.1 \mathrm{M}$ sodium cacodylate for $1 \mathrm{~h}$ at $5^{\circ} \mathrm{C}$. Cells were then pelleted, dehydrated and embedded in Epon. Sections were cut on a Riechert-Jung Ultracut E microtome and stained in aqueous uranyl acetate and lead citrate. Sections were examined in a Phillips CM12 transmission electron microscope.

\section{Acknowledgements}

We thank Francesco Parlati and David Thomas for the S. pombe cnx1 deletion strain and $c n x 1$ expression plasmids and the MRC Laboratory for Molecular Cell Biology for assistance with electron microscopy. The authors would also like to thank Jonathan Knowles for his vision, leadership and support and Stuart Farrow, Jean-Claude Martinou, Kinsey Maundrell, Mark Payton, Roberto Solari and Jurg Tschopp for discussions, support and review of the manuscript.

\section{References}

Ameisen JC (1996) The origin of programmed cell death. Science 272: 1278-1279 Ameisen JC, Idziorek T, Billaut-Mulot O, Loyens M, Tissier JP, Potentier A, and Ouaissi A (1995) Apoptosis in a unicellular eukaryote (Trypanosoma cruzi): implications for the evolutionary origin and role of programmed cell death in the control of cell proliferation, differentiation and survival. Cell Death Differ. 2: $285-300$

Bergeron JJM, Brenner MB, Thomas DY, and Williams DB (1994) Calnexin: a membrane bound chaperone of the endoplasmic reticulum. Trends Biochem. Sci. 19: 124-128

Chen-Levy Z, Nourse J, and Cleary ML (1989) The bcl-2 candidate proto-oncogene product is a 24-kilodalton integral-membrane protein highly expressed in lymphoid cell lines and lymphomas carrying the $t(14 ; 18)$ translocation. Mol. Cell. Biol. 9: $701-710$

Chittenden T, Harrington EA, O'Conner R, Flemington C, Lutz RJ, Evan GI, and Guild BC (1995) Induction of apoptosis by the bcl-2 homologue bak. Nature 374: 733 736

Clarke PGH (1990) Developmental cell death: morphological diversity and multiple mechanisms. Anat. Embryol. 181: 195-213

Cornillon S, Foa C, Davoust J, Buonavista N, Gross JD, and Golstein P (1994) Programmed cell death in Dictyostelium. J. Cell Sci. 107: 26912704

ElderRT, LohEY, and Davis RW (1983) RNA from the yeast transposable element ty 1 has both ends in the direct repeats, a structure similar to retrovirus RNA. Proc. Natl. Acad. Sci. USA 80: 2432-2436

Ellis RE, Yuan J, and Horvitz RH (1991) Mecanisms and functions of cell death. Annu. Rev. Cell Biol. 7: 663-698

Farrow SN and Brown R (1996) New members of the Bcl-2 family and their protein partners. Curr. Op. Gen. Dev. 6: 45-49

Farrow SN, White JHM, Martinou I, Raven T, Pun KT, Grinham CJ, Martinou JC, and Brown R (1995) Cloning of a bcl-2 homologue by interaction with adenovirus E1B19K. Nature 374: 731-733

Garcia I, Martinou I, Tsujimoto Y, and Martinou JC (1992) Prevention of programmed cell death of sympathetic neurons by the bcl-2 proto-oncogene. Science 258 $302-304$

Gonzalez-Garcia M, Perez-Ballestro R, Ding L, Duan L, Boise LH, Thompson CB, and Nunez $\mathrm{G}$ (1994) bcl-XL is the major bcl-x mRNA form expressed during murine development and its product localizes to mitochondria. Development 120 : $3033-3042$

Hengartner MO and Horvitz HR (1994) C. elegans cell survival gene ced-9 encodes a functional homolog of the mammalian proto-oncogene bcl-2. Cell 74: 665-676

Jacobson MD, Burne JF, King NP, Miyashita T, Reed JC, and Raff MC (1993) Bcl-2 blocks apoptosis in cells lacking mitochondrial DNA. Nature 361: 365-369

Kiefer MC, Brauer MJ, Powers VC, Wu JJ, Umansky SR, Tomei LD, and Barr PJ (1995) Modulation of apoptosis by the widely distributed Bcl-2 homologue Bak. Nature 374: 736-739

Korsmeyer SJ, Shutter JR, Veis DJ, Merry DE, and Oltvai ZN (1993) Bcl-2/Bax: a rheostat that regulates an anti-oxidant pathway and cell death. Sem. CancerBiol. 4: $327-332$

Krajewski S, Tanaka S, Takayama S, Schibler MJ, Fenton W, and Reed JC (1993) Investigation of the subcellular distribution of the bcl-2 oncoprotein: residence in the nuclear envelope, endoplasmic reticulum, and outer mitochondrial membranes. Cancer Res. 53: 4701-4714

Maundrell K (1990) nmt1 of Fission Yeast. J. Biol. Chem. 265: 10857 - 10864

Maundrell K (1993) Thiamine-repressible expression vectors pPREP and pRIP for fission yeast. Gene 123: 127-130

Moreno S, Klar A, and Nurse P (1991) Molecular genetic analysis of fission yeast, $S$. pombe. Meth. Enzymol. 194: 795-823

Morgan DO (1995) Principles of CDK regulation. Nature 374: 131-134

Motoyama N, Wang F, Roth KA, Sawa H, Nakayama KI, Nakayama K, Negishi I, Senju S, Zhang Q, Fujii S, and Loh DY (1995) Massive cell death of immature hematopoietic cells and neurons in bcl-x-deficient mice. Science 267: 15061510

Oltvai ZN and Korsmeyer SJ (1994) Checkpoints of dueling dimers foil death wishes. Cell 79: 189-192

Oltvai ZN, Milliman CL, and Korsmeyer SJ (1993) Bcl-2 heterodimerizes in vivo with a conserved homolog, Bax, that accelerates programmed cell death. Cell 74 : 609-619 
Parlati F, Dignard D, Bergeron JJM, and Thomas DY (1995) The calnexin homologue $\mathrm{cn \times} 1^{+}$in S. pombe is an essential gene which can be complimented by its soluble ER domain. EMBO J. 14: 3064-3072

Reed JC (1994) Bcl-2 and the regulation of programmed cell death. J. Cell Biol. 124 $1-6$

Robertson AMG and Thomson JN (1982) Morphology of programmed death in the ventral nerve cord of Caenorhabditis elegans larvae. J. Embryol. Exp. Morph 67: $89-100$

Sambrook J, Fritsch EF and Maniatis T (1989) Molecular cloning: a laboratory manual, 2nd ed. (Cold Spring Harbor Laboratory Press, Cold Spring Harbor, New York)
Veis DJ, Sorenson CM, Shutter JR, and Korsmeyer SJ (1993) Bcl-2 deficient mice demonstrate fulminant lymphoid apoptosis, polycystic kidneys and hypopigmented hair. Cell 75: 229-240

Wellburn SC, Dale C, Ellis D, Beecroft R, and Pearson TW (1996) Apoptosis in procyclic Trypanosoma brucei rhodesiense in vitro. Cell Death Differ. 3: 229236

Williams GT and Smith CA (1993) Molecular regulation of apoptosis: genetic controls on cell death. Cell 74: 777-779

Zhu W, Cowie A, Wasfy GW, Penn LZ, Leber B, and Andrews DW (1996) Bcl-2 mutants with restricted subcellular location reveal spatially distinct pathways for apoptosis in different cell types. The EMBO Journal 15: 4130-4141 